\title{
Human Computer Interaction and medical devices
}

\author{
Chitra Acharya \\ Harold Thimbleby \\ FIT Lab, Future Interaction Technology Laboratory, \\ Swansea University, Swansea, Wales \\ harold@thimbleby.net \\ Patrick Oladimeji \\ chitra.ramnath@gmail.com \\ cspo@swansea.ac.uk
}

\begin{abstract}
To achieve dependable, usable, and well-engineered interactive devices in healthcare requires applied Human Computer Interaction $(\mathrm{HCl})$ research and awareness of $\mathrm{HCl}$ issues throughout the lifecycle, from design through to procurement, training and use. This paper shows that some healthcare devices fall far short, and thus identifies a gap in applied $\mathrm{HCl}$. We use a basic, interactive hospital bed as a case study, arguably so routine and simple enough that there should have been very few problems. However, the bed's interactive control panel design violates standard $\mathrm{HCl}$ principles. It is also badly programmed by the manufacturer. Evidently, something has gone wrong, somewhere from design to procurement, and we argue most of the problems would have been managed or avoided by conventional $\mathrm{HCl}$ processes. Driven by the case study, this paper explores the problems and makes recommendations. There are many similarly poorly designed medical devices. Manufacturers and healthcare purchasing groups should adhere to $\mathrm{HCl}$ processes and guidelines, as well as those provided by regulatory agencies for the design, regulation, and procurement of devices, products, or systems that contribute to patient safety. The challenge is to make $\mathrm{HCl}$ knowledge and priorities available to and effective in this important domain in any places that can make a difference. Eye-tracking, awareness tools, machine learning, coordination, expertise
\end{abstract}

Human Computer Interaction, hospital bed, patient safety, adverse events, medical device design, safety critical design, healthcare.

\section{INTRODUCTION}

Vicente et al. [8] and Johnson et al. [6,18] are some of the few regular contributors to $\mathrm{HCl}$ in healthcare. While deaths from preventable hospital errors have been widely reported [7], the main approaches to improve adverse outcomes (i.e., extended hospital stay, fatality, incorrect treatment) appear to be industrial design and human factors (e.g., [1]) rather than $\mathrm{HCl}$ as such. There have been political perspectives and reviews, such as [5] (which also raises $\mathrm{HCl} /$ security issues), that lament the $\mathrm{HCl}$ problems, and many discussions of human error, notably by Reason [9]. One might assume with the paucity of healthcare $\mathrm{HCl}$ literature that there is no significant problem to be addressed. Yet there are serious avoidable problems in interactive devices in healthcare. Fairbanks and Wears [2] provide a brief but shocking review of usability problems: the lack of research (and lack of research that is taken up) in healthcare is not for the lack of need, but must happen for other reasons. As this paper shows, even beds, apparently trivial and harmless, actually cause deaths due to poor user interfaces. What is surprising is that the design problems seem only to be for want of applied $\mathrm{HCl}$ principles, using principles that we already know. As a piece of $\mathrm{HCl}$ research, the present paper may seem trivial. But here is a place where $\mathrm{HCl}$ could be saving lives, yet we have negligible industry penetration and little impact. In principle, we know the answers to the problems and we know processes to avoid new problems. In this paper, we speculate on the lessons for $\mathrm{HCl}$. Many systems (e.g., electronic patient record systems) and devices so far criticized are complex, and perhaps their poor design can be excused by appealing to complexity. However, in this paper we show that even "trivial" healthcare user interfaces can be badly designed.

\section{THE ROLE OF HCI IN HEALTHCARE}

Adverse incidents in hospitals are under-reported, and for legal, privacy and other reasons are often not discussed widely. Furthermore, the incidents that do reach the research literature are typically discussed from a clinical point of view (for example, how to treat a patient after the incident), and provide few insights into human factors or $\mathrm{HCl}$ issues related to 
the incident. However, some thorough retrospective analyses of incidents have been published, notably the root cause analysis of a drug-overdose related fatality, which included a professional Human Factors (HF) study of the user interface [4]. The HF study of the infusion pump took four hours with a total of six participants. The study revealed major Human Factors problems, and concluded by saying “... lack of insight into design issues is very common given that the healthcare world is filled with these issues and healthcare personnel are rewarded for working around them with little complaint." There were many explicit $\mathrm{HF} / \mathrm{HCl}$ criticisms in the root cause analysis, including criticisms of contributions to the fatality other than the interactive device design itself, such as poor labeling. In our view, that a four hour study can so readily highlight such serious problems raises concerns that the manufacturer either failed to do any usability analysis of its product, or it did but (for some reason) ignored or was unable or unwilling to use the insights from its own studies. Certainly, in an ideal world, one would imagine that manufacturers would routinely invest more in usability than the negligible cost of the brief study done as part of the root cause analysis - the insights of which came too late. Evidently, for this device, the nurses who used it, and for the patient who died through its use, some things went seriously wrong. Equally, it seems a concern that a study of the sort done in the root cause analysis was not performed routinely as part of procurement. The broad implication is that $\mathrm{HCl}$ work does not usefully contribute to the design, procurement or user training (the root cause analysis also identified weaknesses here) of devices. In this paper we will take a bed as a case study. The bed has not, to our knowledge, caused any adverse incidents or even any that have led to root cause analyses, but the general impressions to the drug dose fatality are similar, and one cannot argue, say, that "beds are complicated to get to work at all, so $\mathrm{HCl}$ should take second place." On the contrary, beds are simple and, as we show, $\mathrm{HCl}$ can easily contribute to improving them. The concern is that the bed has avoidable $\mathrm{HCl}$ defects (which we detail below); we will argue that the defects could have been avoided by appropriate use of $\mathrm{HCl}$ either during design or during procurement (i.e., either so the bed was better designed in the first place, or so that the hospital obtained the $\mathrm{HCl}$ evidence to avoid purchasing it). It is useful to break down $\mathrm{HCl}$ into four different ways that it can contribute to healthcare:

- Design processes: including evaluation, iterative design, regulatory approval, and procurement. Such issues are covered in various standards, such as ISO13047.

- Industrial design: including ergonomics, human factors, and design.
- Interaction programming: including the hidden complexity of interaction, such as state, properties, invariants, modes. The programming of user interfaces is rarely covered in the $\mathrm{HCl}$ literature [12].

- Teaching and dissemination to the community: teaching $\mathrm{HCl}$ requires students to engage with the subject, and typically involves critiques, developing and evaluating prototypes, and learning $\mathrm{HCl}$ evaluation methods. The healthcare domain raises serious ethical issues: patients are a vulnerable group, patient data is restricted, infection control and other issues limit access, liability issues for device manufacturers and users limit the availability of detailed and accurate device error reports, and medical devices are very expensive - and they are hard to understand without clinical experience. So students typically end up working with easier to access devices, such as mobile phones and domestic appliances and ideas that, while fashionable, are not so important in safety critical domains.

It seems that $\mathrm{HCl}$ in healthcare is not fundamentally different requiring (for instance) new research; what it needs is a higher priority and easier access for students, industry and researchers.

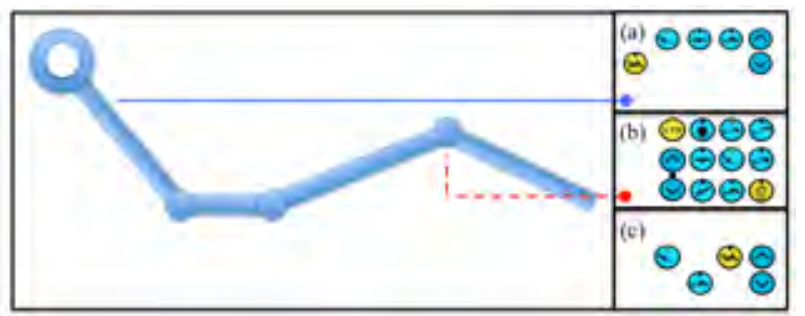

Figure 1. Hospital bed having (a) Nurse (b) Attendant and (c) Patient control panels (Simulation snapshot).

Without engaged teaching, manufacturers and hospitals are unlikely to be able to recruit staff with $\mathrm{HCl}$ skills and knowledge, so $\mathrm{HCl}$ problems in healthcare will persist.

\section{BEDS AND ADVERSE EVENTS}

In 1999, the FDA(USA Food and Drug Administration) formed the Hospital Bed Safety Workgroup, to improve the safety of hospital beds. The workgroup is developing resources including dimensional guidelines, measurement tools, and educational materials to assist manufacturers, caregivers and consumers [17]. Between 1985 and 2008, the FDA received 772 incidents of patients caught, trapped, entangled or strangled in hospital beds. The reports included 460 deaths, 136 nonfatal injuries, and 176 cases where staff needed to intervene to 
prevent injuries. Most patients were frail, elderly or confused. Larry Kessler of the FDA Office of Science and Engineering Laboratories [16] said "While these numbers appear small, we believe they are signals about significant adverse events. Often, adverse events such as these go unreported to the FDA, making it likely our counts of these tragic adverse incidents are lower than the number that actually occurs." MHRA (UK Medicines and Healthcare products Regulatory Agency) publishes alerts to warn healthcare professionals about poorly-designed malfunctioning hospital beds [15]. The BBC (British Broadcasting Corporation) has also reported hospital beds being linked with injury and death [14].

\section{AN ILLUSTRATIVE CASE STUDY}

We use a basic hospital bed as a case study in this paper. Our case study is an actual, typical basic electric profiling modern hospital bed, of which we have made an accurate interactive web-based model. While we have taken great care to make our study correct and accurate, we do no disclose the manufacturer or bed model; the wider issues are what we are concerned with, rather than specifics of this bed which we take to illustrate the wider issues. Beds made by other manufacturers are comparable, and other hospital procurement, nurse training, and so forth are all comparable. Nevertheless our methodology [11] calls for an accurate simulation of the device, so that our discussion, evidence and claims are justifiable. The full simulation of the hospital bed control panels is available at http://cs.swan.ac.uk/ cschitra. The hospital bed simulation has approximately 1,600 lines of Adobe Flex ActionScript code (though its size is of no consequence in use). Although for our study we built a simulation, note that the manufacturers themselves would not need to build one since they must already have the source code of the actual device; however the discipline of reverse engineering, as argued in [11], has usefully helped reveal design issues that may have been missed in a less systematic approach. The bed has three control panels: one each for Nurse, Attendant, and Patient, illustrated in Figure 1, which adjust the bed to change the patient's posture. Figure 2 shows a close-up of the panels and their buttons. The images on the hospital bed control panels and their corresponding labels are illustrated in Figure 3. There are duplicate panels on each side of the bed. Whatever the bed configuration, if the patient requires cardiopulmonary resuscitation (CPR) it is crucial for the bed to harden and flatten quickly at the touch of a button [14]. There is a special CPR button to do this.

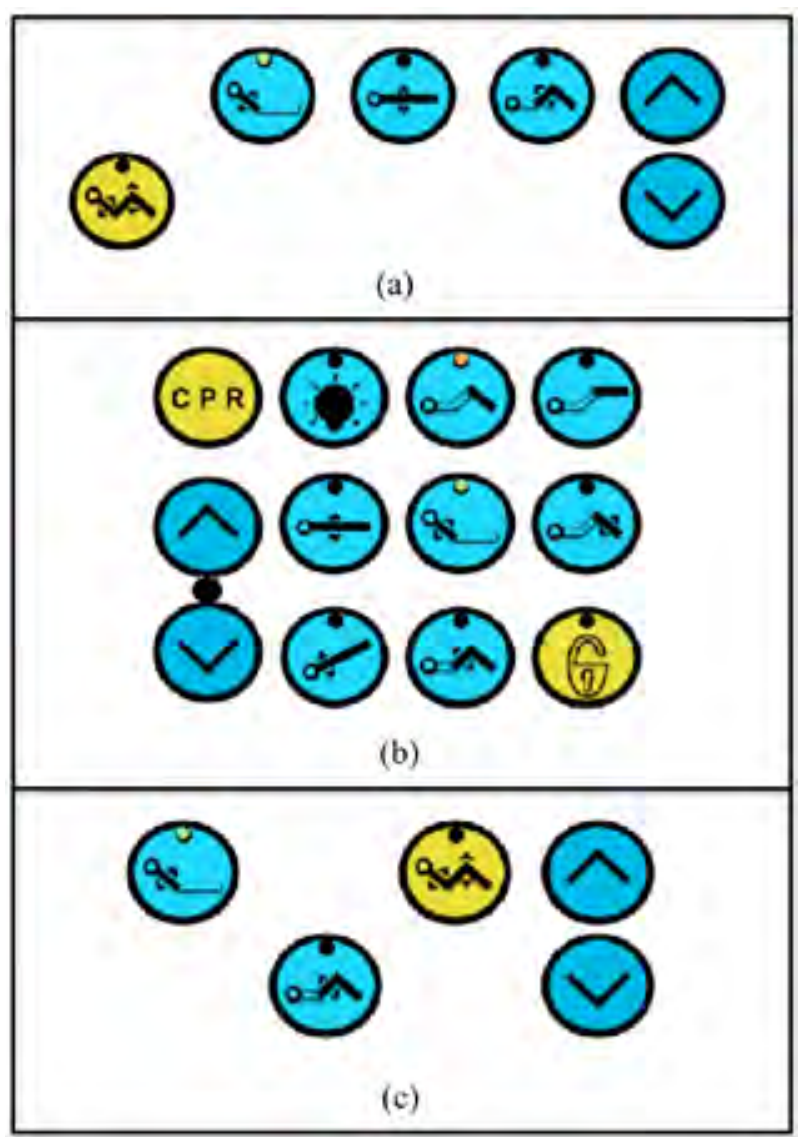

Figure 2. Simulation snapshot of the bed control panels. (a) Nurse (b) Attendant and (c) Patient control panels.

During our study we found we could crash the bed's software and nothing, including the CPR button, would work. (Although a "crash" is caused by a programming problem it does result in an $\mathrm{HCl}$ problem: dismissing crashes as programming, as some of our colleagues have suggested, would be a bit like dismissing, say, screen design as painting and no of concern to $\mathrm{HCl}$.) Furthermore, the control panel design violates routine $\mathrm{HCl}$ principles. The bed itself has a wide range of attractive features for hospitals: it has split safety sides with embedded user controls, function lockouts, automatic CPR mode with dual-sided manual CPR levers, as well as clinical features such as a low height to reduce falls, profiling system for body pressure reduction, infection control design for cleaning and decontamination, maneuverability ... and so on.

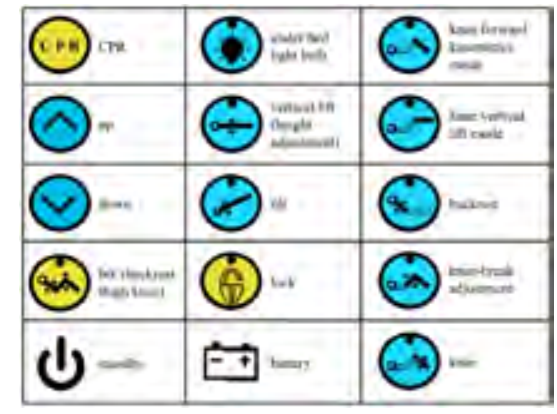

Figure 3. Images on the hospital bed control panels. 
Figure 1 schematically illustrates the bed, along with its control panels used to adjust it. The bed is targeted at general wards; in intensive care units, pediatrics and elsewhere, more sophisticated beds are used - which display the user interface design issues we discuss in this paper, but perhaps camouflaged by their wider range of facilities. Thus the study of a "simple" bed has many advantages for the purposes of this paper. It is possible to be opinionated about user interface design and unrepresentative of user communities. For some questions, we therefore asked 10 participants for their views. Ten is a small number, but the wide variation in opinions between the participants suggests further studies are called for. Conversely, it suggests how cheap informative pilot user surveys can be as a form of discount usability [3], and also raises questions about tradeoffs between ease of use and manufacturing costs. However, the variations in opinion notwithstanding, mapping, feedback, and confusion errors made the device difficult to use for most of the participants. Following the "interaction walkthrough" method [11], we studied the bed carefully and programmed an accurate simulation of the bed. This method produces an unusually detailed analysis of an interactive device, namely a thorough reverse engineering, and may be considered a rigorous form of expert analysis and cognitive walkthrough.

\section{UI SIMULATION}

The three control panels (Nurse, Attendant, and Patient) were reverse engineered and a program was written to closely simulate the behavior of the bed as a graphical user interface. A simulation snapshot of the hospital bed control panels is illustrated in Figure 2. A UI discovery tool [13] was used to obtain the state transition diagrams (Figure 4 and Figure 5). The $\mathrm{UI}$ discovery tool works by monitoring variables in the system under discovery while the user interface buttons are systematically and automatically clicked until all states in the system has been explored.

The bed can be modeled as a state transition system. For example, exploring the BACKREST part of the bed using the UP and DOWN buttons produces 13 states, as illustrated in Figure 4. In our model, the backrest moves in steps of five degrees (an approximation of a continuous movement) from zero to sixty degrees.

The arrows labeled Up, Up0, and Up1 are related to the replication of the UP button on all the three panels (Nurse, Attendant, and Patient). Similarly, the three arrows labeled Down, Down0, and Down1 are related to the replication of the DOWN button on all the three panels. Note that the duplicated control panels are not simulated.

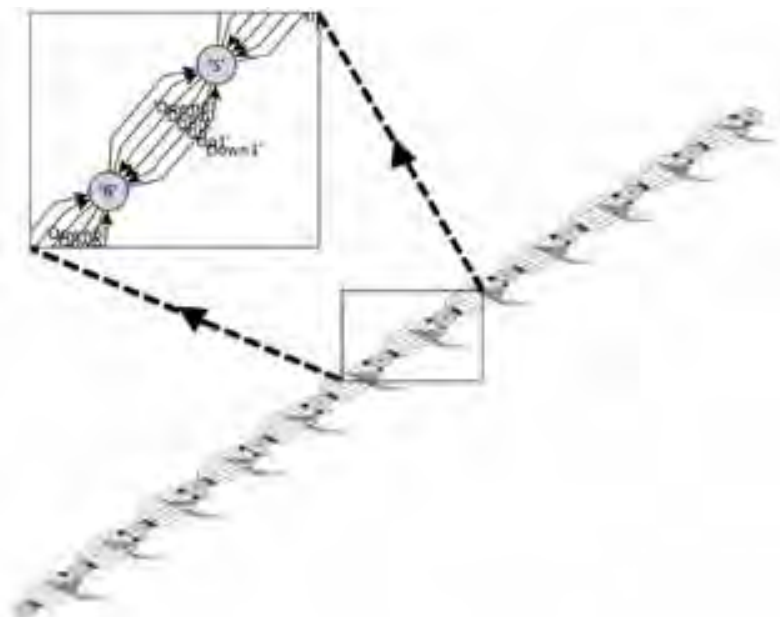

Figure 4. State transition diagram (with 2 states shown in detail) for BACKREST, UP and DOWN buttons is 13 states.

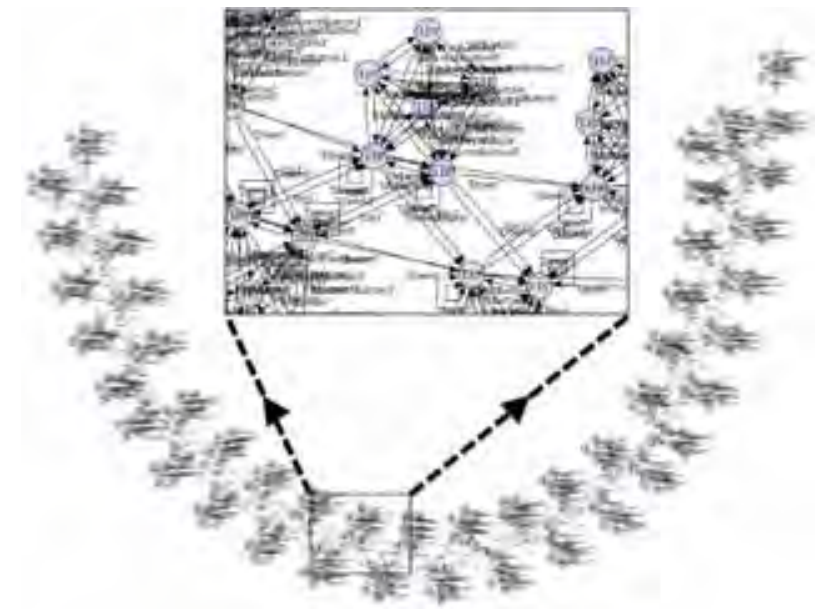

Figure 5. State transition diagram for BACKREST, LIFT, TILT, UP and DOWN buttons is 276 states. This diagram is only intended to illustrate the complexity of the device

for this paper; in normal use, the diagram would be viewed in a zoomable user interface and any detail can be examined clearly.

The simulation incorporates the timing issues related to LOCK, BACKREST, THIGH, KNEE, TILT, LIFT, and BTK (backrest thigh knee) buttons, but the state transition system obtained does not reflect the timing issues, as the discovery process was set to ignore them. If we include two more buttons, TILT and LIFT (height adjustment button), this results in 276 states, as illustrated in Figure 5. The tilt moves in steps of 1 degree (an approximation of a continuous movement) from zero to 10 degrees. The height adjustment is also an approximation of a continuous movement. There are various ways of visualizing and analyzing state machines, but which are beyond the scope of the present paper to explore. Apart from the major defects in the design (e.g., crashing) the analysis of the state space of the bed itself is relatively unrevealing with respect to $\mathrm{HCl}$ issues. 


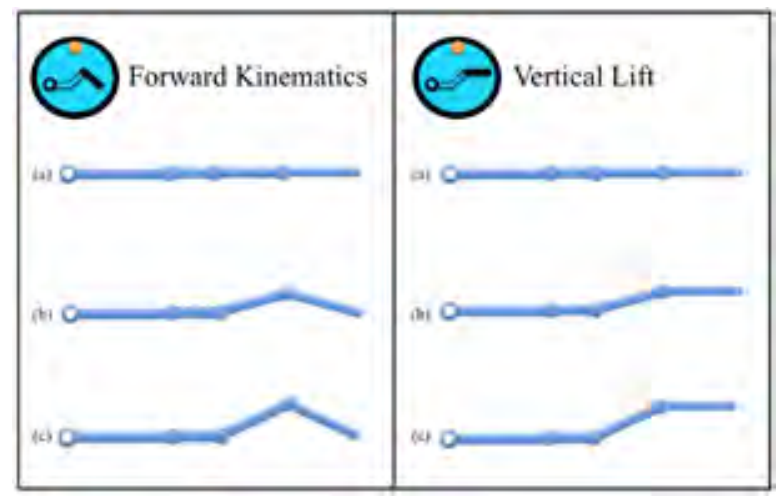

Figure 6. Knee-break adjustment has two modes forward kinematics and vertical lift modes. (a) Initial (b) intermediate and (c) final postures.

\section{CONVENTIONAL HCI PRINCIPLES}

The design of the hospital bed control panel violates well-known and conventional $\mathrm{HCl}$ principles. The main principles that are violated are discussed here, specific to how the bed works. 6.1 Error recovery After a crash, no buttons worked, and particularly worrying, the CPR button did not work. There were no suggestions for error recovery. The users would have to deploy manual CPR levers or they would have to reset the software (by disconnecting the bed from the mains supply). It is unlikely that a nurse under pressure to perform a CPR would undertake either course of action reliably.

\subsection{Appropriate affordance}

The standby symbol is an example of a false affordance. It should be either a switch or a button. The participants pressed the symbol and nothing happened. They then realized it was not a button; it is a graphical symbol for the Standby LED. This design issue confused participants.

\subsection{Modes and hidden state}

The knee-break adjustment has two different modes, forward kinematics and vertical lift modes (Figure 6) that affect the way the knee-break section moves when the THIGH or KNEE or BTK button is pressed. The BTK button adjusts the backrest, thigh and knee postures; which could also be done using a combination of BACKREST, THIGH and KNEE button presses.

\subsection{Mapping}

The mapping of images on attendant control panel (located on one of the sides of the bed), illustrated in Figure 7 (simulation snapshot) confused many participants as the images on the buttons (circled in red) are mirror images of the physical bed.

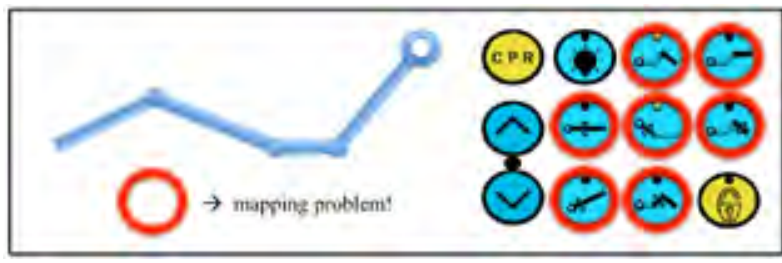

Figure 7. User's workload is increased due to bad mapping of images on attendant control panel (buttons circled in red) located on one of the sides of the bed (simulation snapshot).

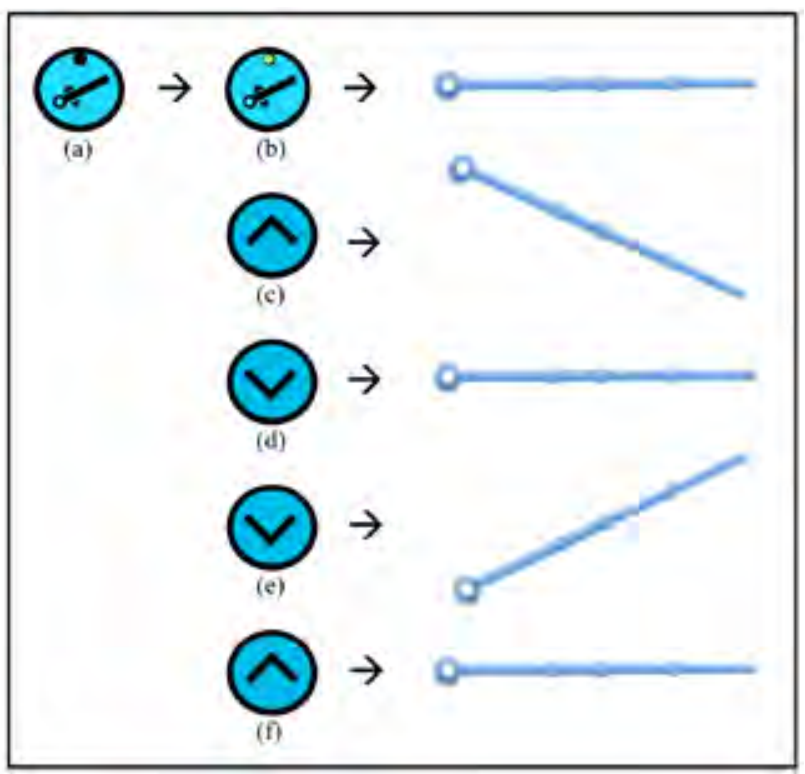

Figure 8. Mapping confusion with TILT button as pivot is at the centre.

The mapping of a few buttons confused many participants. Press the TILT button (Figure 8a). The green LED glows indicating TILT button (Figure 8b) is selected. Then press UP button (Figure 8c), to get the desired tilt posture. Then press DOWN button (Figure 8d), the bed becomes parallel to the floor. Continue pressing the DOWN button (Figure 8e) and we can observe that the pivot is at the centre (simulation snapshot). The mapping for TILT button confused many participants.

\subsection{Feedback}

Battery LED is an example of bad feedback as illustrated in Figure 9. When the device is connected to a power source, the Standby LED glows indicating the device is functional (Figure 9: State A). When the device is not connected to a power source, both standby and battery LEDs do not glow (Figure 9: State B). But the device is functional and can be operated on battery backup. This illustrates bad feedback of battery LED and thus increases user's workload. When the user continues to operate the device on battery backup, there is no indication if the battery drains out. When it is connected to a power source, both standby and battery LEDs glow (Figure 9: State $\mathrm{C}$ ); indicating the device is functional and battery is charging. Once the battery is fully charged, the battery LED stops glowing (Figure 9: State A). 


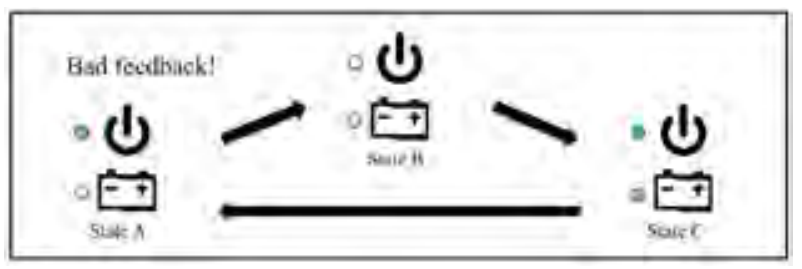

Figure 9. User's workload is increased due to bad feedback from battery LED (State $B$ ).

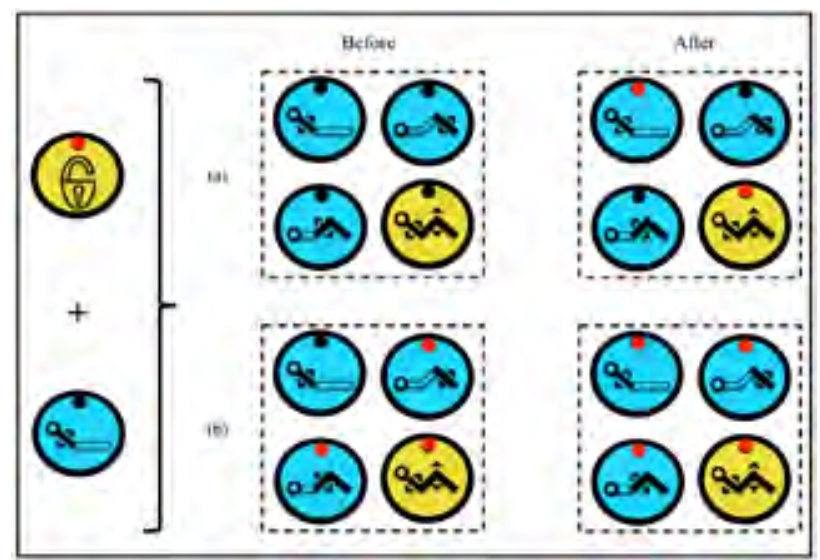

Figure 10. Function lockouts of inter-related buttons: locking BACKREST button: (a) THIGH and KNEE buttons unlocked(b) THIGH and KNEE buttons locked.!

\subsection{Confusion errors}

More than one person trying to interact with the bed from different control panels could lead to confusion. There may be an argument between a nurse and a patient on the appropriate posture of the bed. Function lockouts of inter-related buttons (Figure 10, Figure 11, Figure 12, Figure 13, Figure 14 and Figure 15) may also possibly lead to confusion. When the THIGH button is locked, the KNEE button gets locked (or vice versa) - illustrated in Figure 11 and Figure 12. Consequently, it also locks the BTK button (if not already locked - Figure 11a and Figure 12a). When the BACKREST button is locked, it also locks the BTK button (if not already locked Figure 10a). When the THIGH button is unlocked, the KNEE button gets unlocked (or vice versa) illustrated in Figure 14 and Figure 15 - and unlocks the BTK button (only if the BACKREST button is not locked-Figure 14a and Figure 15a). Similarly, when the BACKREST button is unlocked, the BTK button gets unlocked (only if both the THIGH and KNEE buttons are unlocked - Figure 13a). This can be very confusing and burdens the user to remember the function lockout of inter-related buttons (also bearing in mind the lockout can be initiated by any one of the three control panels).

\subsection{User workload}

The attendant control panel mapping problem, battery LED bad feedback, and other problems discussed above, confused many participants, which could possibly increase the user's cognitive workload.

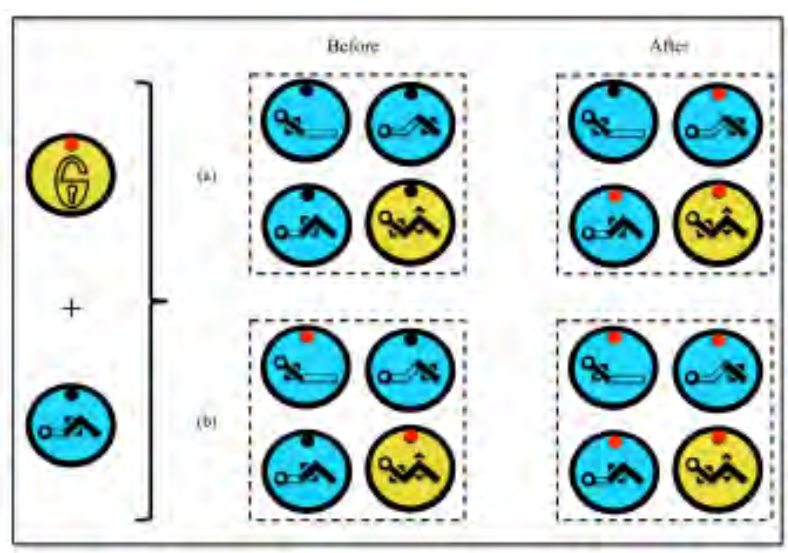

Figure 11. Function lockouts of inter-related buttons: locking THIGH button: (a) BACKREST button unlocked (b) BACKREST button locked.

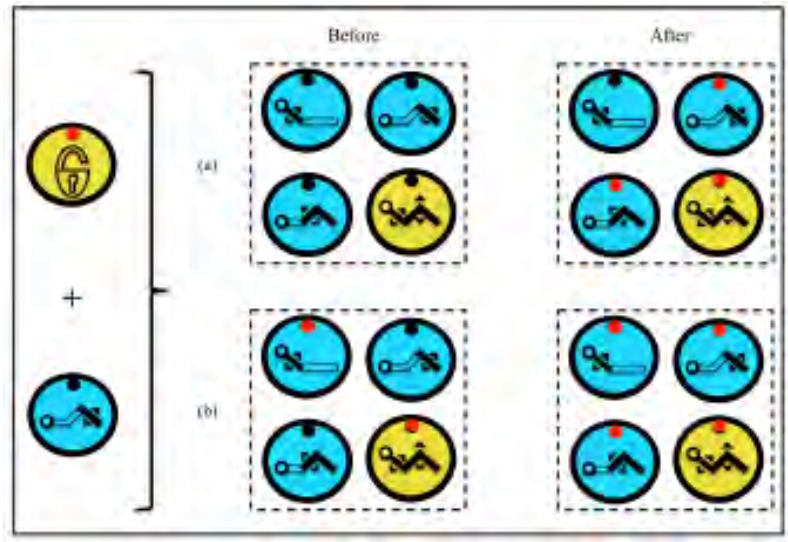

Figure 12. Function lockouts of inter-related buttons: locking KNEE button: (a) BACKREST button unlocked (b) BACKREST button locked.

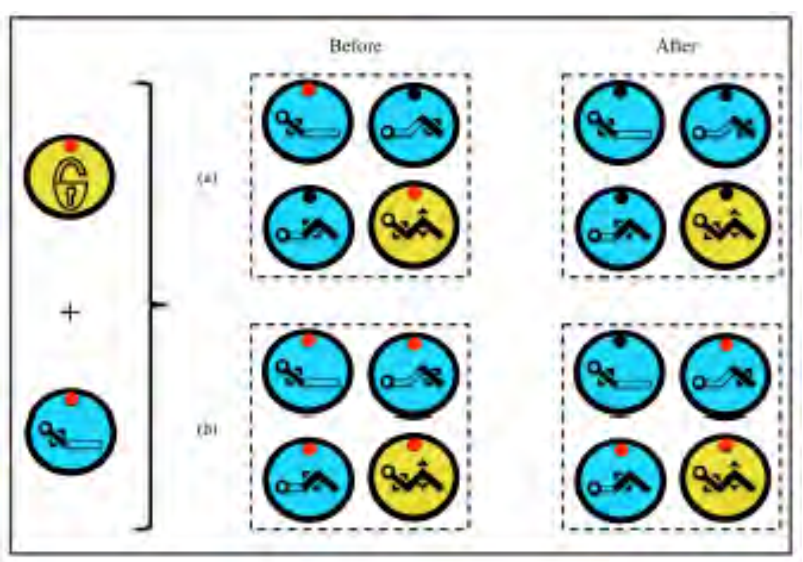

Figure 13. Function lockouts of inter-related buttons: unlocking BACKREST button: (a) THIGH and KNEE buttons unlocked (b) THIGH and KNEE buttons locked. 


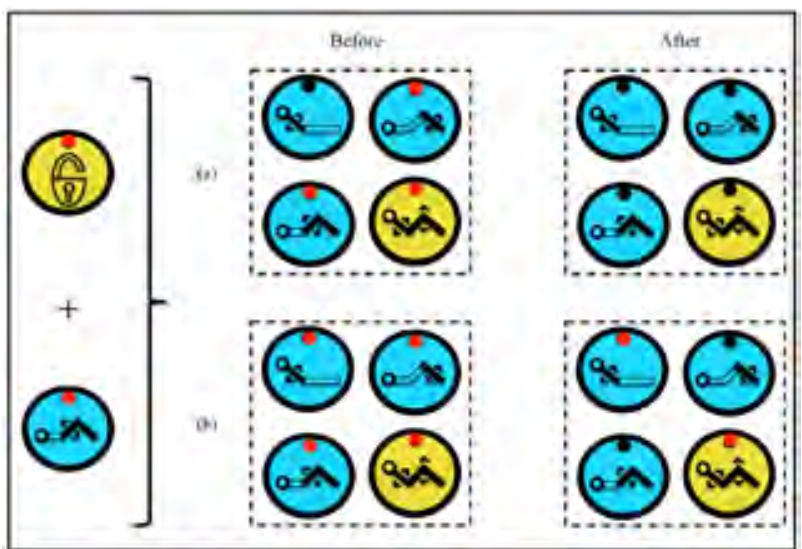

Figure 14. Function lockouts of inter-related buttons: unlocking THIGH button: (a) BACKREST button unlocked (b) BACKREST button locked.

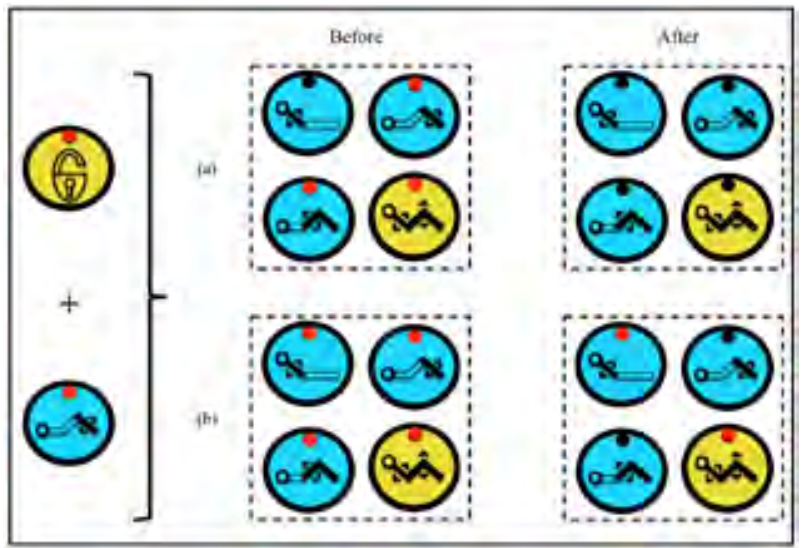

Figure 15. Function lockouts of inter-related buttons: unlocking KNEE button: (a) BACKREST button unlocked (b) BACKREST button locked.

\section{PROPOSED DESIGN \& VALIDATION}

The authors are collaborating with design team, medical school, users and manufacturers to redesign the hospital bed, by applying $\mathrm{HCl}$ and human factors and fixing programming bugs. The proposed design is expected to reduce confusion and user's cognitive workload. The proposed design consists of the following:

- Correct mapping of the images on the buttons of all the control panels.

- Replacement of the image on the TILT button with the correct image to provide mapping that is easily learned and readily recalled. The TILT button illustrated in Figure 16:(a) and/or (b) could be less confusing to the participants.

- Good feedback of battery LED, such that when the device is not connected to a power source, if the battery LED glows, it will indicate presence of battery backup (illustrated in
Figure 17: State B') and blinking LED will suggest that battery backup is not sufficient and it has to be connected to a power source.

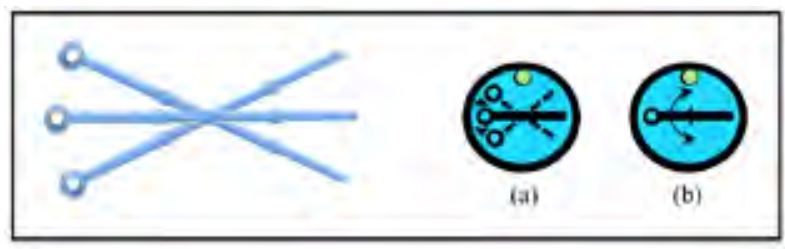

Figure 16. Mapping confusion with TILT button as pivot is at the centre. TILT button (a) and/or (b) could be less confusing to the participants.

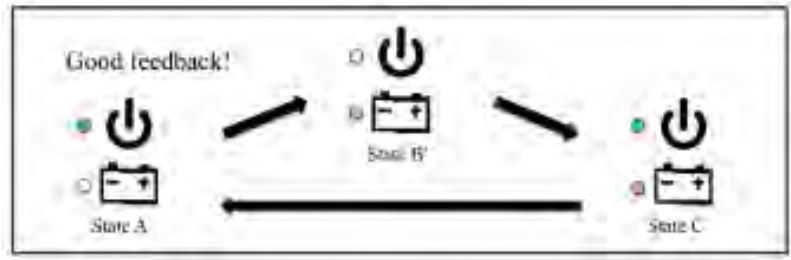

Figure 17. User's workload could be decreased by good feedback from Battery LED (State B').

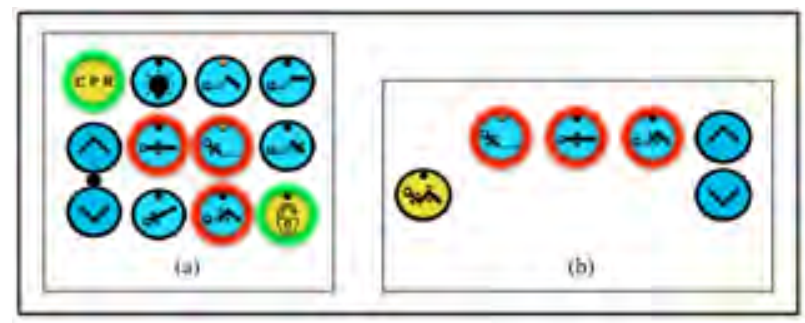

Figure 18. (a) Attendant and (b) nurse control panels have three buttons (BACKREST, LIFT and THIGH buttons) in common (circled in red). Nontrivial buttons (CPR and LOCK buttons) are located on the attendant control panel (circled in green).

- Integrate attendant and nurse control panels (BACKREST, LIFT and THIGH buttons in common; Figure 18, circled in red) into one superset control panel. Locate non-trivial buttons - CPR and LOCK buttons (Figure 18, circled in green) - on this superset control panel.

- Not use hidden or obscure modes for kneebreak adjustment mode and group interrelated function lockout buttons.

- Avoid false or misleading affordances. Example, the Standby symbol should be on a button.

- Help users to easily recognize, diagnose, and recover from errors by providing alarms and other error feedback which are precise, indicating the problem and perhaps constructively suggesting a solution. 
The authors are planning to conduct an empirical study to compare experimentally the new prototype with the existing commercially available interface. The study aims at validating the acceptance of the new prototype. In this study, the users will be made to change the bed postures on both the existing and new interfaces. It is expected the new prototype will eliminate errors and improve the usability of the bed.

\section{CONCLUSIONS}

The influential National Academies report [7] flagged serious concerns about preventable error in healthcare; the more recent NRC report [10] further emphasized the role of user models and $\mathrm{HCl}$ in solutions in healthcare. We believe that without more attention to $\mathrm{HCl}$, and the practical difficulties of raising its profile, healthcare will continue to be problematic. If our bed case-study is anything to go by, the issue of usability testing and usability evaluation for healthcare devices appears to be overlooked and under-rated - particularly considering the amount of standard $\mathrm{HCl}$ design problems present in many devices in use in hospitals (cf $[2,4-7,10,11,15-$ 18]). As a case study, the value of research in $\mathrm{HCl}$ principles and practices was investigated using a modern interactive hospital bed. The bed has three control panels, for attendant, nurse, and patient, which adjust the bed to change the patient's posture. If the patient requires CPR it is crucial for the bed to flatten quickly, and there is a special CPR button to do this. During our study we found we could crash the bed's software/firmware and nothing, including the CPR button, would work. Furthermore, the control panel design violated basic $\mathrm{HCl}$ principles creating mapping, feedback, and confusion errors. More generally than a case study, this paper has identified a gap in $\mathrm{HCl}$ : that is, in healthcare, user interfaces ought to be dependable, usable and well-designed but they are not! As our introduction made clear, the bed is not a special case, but appears to be typical in this regard. What is unusual is the simplicity of the bed and the regret that for such a simple device it ought to have been very easy to make it of high quality. If we can't do beds, what hope is there for infusion pumps, ventilators and even electronic patient records? Device manufacturers and healthcare purchasing groups should be aware of and adhere to $\mathrm{HCl}$ processes and guidelines, as well as those guidelines provided by regulatory agencies for the design and procurement of devices, products, or systems that contribute to patient safety. The challenge is to make $\mathrm{HCl}$ knowledge and priorities available to and effective at the right points in this important domain.

We suggest the following points need to be seriously considered by the $\mathrm{HCl}$ community:
- The application of $\mathrm{HCl}$ seems to be ineffective in a significant safety-critical domain, healthcare. It appears to be fully or partly absent from every step, through design, development, regulatory bodies, marketing, procurement, training, use, ... until it is too late.

- $\mathrm{HCl}$ practitioners appear to be avoiding an important area; equally, the people working in the area seem unaware of $\mathrm{HCl}$. For example, are computer science departments graduating developers unaware of $\mathrm{HCl}$ ? Perhaps design / manufacturers / regulators / procurement may not even be using qualified people. If we are to improve the quality of safety-critical areas then $\mathrm{HCl}$ has to be a leader in exposing problems and showing it can avoid or manage defects.

- There is a tiny literature on $\mathrm{HCl}$ in safetycritical areas, particularly in healthcare. In the healthcare domain, most of the literature concentrates on clinical sequelae of adverse events (e.g., by administering CPR and an antidote, the patient recovered from a drug overdose), not on the design or $\mathrm{HCl}$ issues (e.g., the ergonomics of the keypad encouraged the nurse to enter the wrong drug dose, and the user interface design provided no active review of the dose) that created the latent conditions for the error. In the USA, the mandatory medical device error reports often blame the user whenever no technical malfunction could be detected. The user is expected to know how to use the device, no matter how poorly designed its interface. $\mathrm{HCl}$ avoiding healthcare may be for legal reasons, but it means there is very little learning and background knowledge for manufacturers, procurement, and regulatory bodies.

- $\mathrm{HCl}$ rarely discusses error and negative outcomes; much of the literature is about positive developments. While this paper discussed a single case study that seems to generalize to a worrying state about $\mathrm{HCl}$ in healthcare generally, in fact it did not rigorously establish that all or most devices in all or most hospitals have poor $\mathrm{HCl}$. Moreover there is no analogous literature (say, for infusion pumps) that does so either.

Additionally, points can be made about the contributions of healthcare to the usability of devices: 
- Near misses are rarely reported or acted on. Typically, an incident is only reported if it results in an adverse clinical event. For example, in the operating theater we have seen devices crashing and being rebooted: this has become normal practice, and is not reported (provided there is no immediate clinical outcome).

- Adverse events are rarely explored from any perspectives other than their clinical implications. For example, how a patient is treated after an overdose is a clinical issue, but the $\mathrm{HCl}$ and latent conditions leading to the incident are generally ignored.

One of the possibilities is that $\mathrm{HCl}$ is complex and that medical devices and environments are complex. One might also add further excuses such as the highly competitive market, rapidly changing technologies, and problems undertaking reliable ecological user studies (e.g., patient confidentiality, or largely irrelevant medical research standards such as randomized control trials). On the contrary, this paper showed that basic $\mathrm{HCl}$ can contribute to the healthcare.

Of course some problems of safety critical systems lie outside $\mathrm{HCl}$ itself; perhaps business models within a regulatory framework that ignores $\mathrm{HCl}$. It is clearly important to consider how we train and motivate $\mathrm{HCl}$ practitioners to engage - or avoid - important areas such as healthcare.

\section{ACKNOWLEDGMENT}

This research was funded by EPSRC Grants EP/ F020031/1 and EP/G059063/1.

\section{REFERENCES}

[1] Department of Health and Design Council. 2003. Design for Patient Safety: A system-wide design-led approach to tackling patient safety in the NHS. London: Department of Health Publications. [2] Fairbanks, R. J. and Wears, R. L. 2008. Hazards with medical devices: The role of design. Annals of Emergency Medicine, 52(5):519-521.

[3] Gray, W. D., et al. 1995. Discount or disservice? Discount usability analysis - evaluation at a bargain price or simply damaged merchandise? In ACM CHI '95, pages 176-177.

[4] ISMP Canada. 2007. Fluorouracil Incident Root Cause Analysis. Toronto: Institute for Safe Medication Practices Canada.

[5] Johnson, C. W. 2009. Politics and patient safety don't mix: Understanding the failure of large-scale software procurement in healthcare. In Proceedings IET System Safety 2009.

[6] Johnson, T. R., et al. 2005. The role of patient safety in the device purchasing process. In Henriksen, K., et al., editors, Advances in Patient Safety: From Research to Implementation, pages 341-352. MD: Agency for Healthcare Research and Quality.

[7] Kohn, L. T., et al., editors. 2000. To err is human: Building a safer health system. Washington, D.C.: National Academy Press.

[8] Lin, L., Vicente, K. J. and Doyle, D. J. 2002. Patient safety, potential adverse drug events, and medical device design: A human factors engineering approach. Journal of Biomedical Informatics, 34:274-284.

[9] Reason, J. 1992. Human Error. Cambridge University Press, Cambridge, UK.

[10] Stead, W. W. and Lin, H. S., editors. 2009. Computational Technology for Effective Health Care: Immediate Steps and Strategic Directions. Committee on Engaging the Computer Science Research Community in Health Care Informatics, National Research Council.

[11] Thimbleby, H. 2007. Interaction walkthrough: Evaluation of safety critical interactive systems. In Doherty, G. and Blandford, A., editors, DSVIS 2006, volume 4323 of LNCS, pages 52-66. Springer Verlag.

[12] Thimbleby, H. 2007. Interaction Programming, MIT Press.

[13] Thimbleby, H. and Oladimeji, P. 2009. Social network analysis and interactive device design. In Calvary, G., Graham, T. C. N. and Gray, P., editors, ACM SIGCHI Symposium on Engineering Interactive Computing Systems, EICS'09, pages 91-100.

[14] UK BBC (British Broadcasting Corporation). 2010. Archive. http://www.bbc.co.uk/archive/index. shtml.

[15] UK Medicines and Healthcare products Regulatory Agency (MHRA). 2010. Medical Device Alerts. http://www.mhra.gov.uk/Publications/ Safetywarnings/Medic alDeviceAlerts.

[16] USA Food and Drug Administration (FDA). March 9 2006. News Release: FDA Issues Guidance on Hospital Bed Design to Reduce Patient Entrapment. http://www.fda.gov/NewsEvents/Newsroom/ PressAnnounce ments/2006/ucm108612.htm.

[17] USA Food and Drug Administration (FDA). 2010. Hospital Bed Safety Workgroup. http://www. fda.gov/cdrh/beds/.

[18] Zhang, J., Johnson, T. R., et al. 2003. Using usability heuristics to evaluate patient safety of medical devices. Journal of Biomedical Informatics archive, 36(1/2):23-30 